\title{
AUTOMATIC ATTENTIONAL SHIFTS BY GAZE, GESTURES, AND SYMBOLS
}

\author{
Wataru SATO $^{1)}$, Takanori KOCHIYAMA ${ }^{2)}$, Shota UONO ${ }^{1)}$, and Sakiko YOSHIKAWA ${ }^{1)}$ \\ ${ }^{1)}$ Kyoto University, Japan, ${ }^{2)}$ Advanced Telecommunications Research Institute International, Japan
}

\begin{abstract}
Previous studies suggest that attention shift can be automatically triggered commonly by gaze and hand gestures of other individuals and symbols, but this idea remains controversial. We investigated this issue with two experiments using a cuing paradigm. The non-predictive cue was centrally presented before target presentation in the periphery. The cues were photographic stimuli indicating averted or neutral directions, which were expressed by eyes, a hand, or an arrow. Valid cues consistently shortened the reaction time needed to localize the targets compared with invalid or neutral cues for all three types of stimuli. These results suggest a common psychological mechanism for automatic attentional shift triggered by gaze, gestures, and symbols.
\end{abstract}

Key words: arrows, attentional shift, gaze, hand gestures

Sharing attention with others is an indispensable psychological ability for humans. It allows individuals to share critical information on the environment and to respond appropriately in social coordination. Experimental studies demonstrate that human infants (Csibra, 2003) and monkeys (Emery, Lorincz, Perrett, \& Oram, 1997) can follow the gaze of other individuals, suggesting an evolutionary basis for this mechanism.

Previous studies have revealed that another individual's eye direction automatically triggers covert shifts of attention (for a review, see Frischen, Bayliss, \& Tipper, 2007). For example, Friesen and Kingstone (1998) presented a central gaze cue that was expressed by the eye direction of a schematic face. The participants' reaction time (RT) to detect, localize, or identify a target was shorter when the target was preceded by a valid gaze cue than when it was preceded by an invalid or neutral one. An attentional shift occurs even when the cue is unpredictive of the target location. Some studies have also revealed that an attentional shift occurs when the cue is counterpredictive of the target location (Driver, Davis, Ricciardelli, Kidd, Maxwell, \& Baron-Cohen, 1999; Friesen,

Wataru Sato, Department of Comparative Study of Cognitive Development (funded by Benesse Corporation), Primate Research Institute, Kyoto University. Takanori Kochiyama, Brain Activity Imaging Center, Advanced Telecommunications Research Institute International. Shota Uono and Sakiko Yoshikawa, Department of Cognitive Psychology in Education, Kyoto University. Sakiko Yoshikawa, Kokoro Research Center, Kyoto University.

We thank Dr. A. Nakamura for technical support, and Professors M. Matsumura and A. Miyake for helpful advice. This study was supported by Special Coordination Funds for promoting Science and Technology from The Science and Technology Agency of the Japanese Government.

Correspondence should be directed to Wataru Sato, Department of Comparative Study of Cognitive Development (funded by Benesse Corporation), Primate Research Institute, Kyoto University, Kanrin, Inuyama, Aichi 484-8506, Japan (E-mail: sato@pri.kyoto-u.ac.jp). 
Ristic, \& Kingstone, 2004). A recent study further indicated that the attentional shift by gaze occurs without conscious awareness of the gaze (Sato, Okada, \& Toichi, 2007). These data suggest that the gaze-triggered attentional shift is automatic.

Some previous studies reported that other centrally presented cues, such as arrows, trigger attentional shifts only when the participants intentionally follow the direction of the cues (e.g., Posner, 1980). Based on such data, the attentional shift triggered by gaze cues has been proposed to be special (e.g., Friesen \& Kingstone, 1998).

However, recent studies reported that an automatic attentional shift could be triggered by centrally presented arrows (Hommel, Pratt, Colzato, \& Godijn, 2001; Ristic, Friesen, \& Kingstone, 2002; Tipples, 2002). These data raise a question regarding the special status of eyes as attention-triggering stimuli. However, this issue remains controversial (cf. Frischen et al., 2007). For example, Friesen, Ristic, and Kingstone (2004) reported negative results regarding automatic attentional shifts in response to arrows. Friesen et al. speculated that different experimental conditions may produce different results. Further evidence would be required about whether the attentional shift could be automatically triggered by arrows. Specifically it would be useful to compare the patterns of effects of eyes and arrows.

In addition to these cues, other lines of research suggest that hand-pointing gestures may automatically trigger attentional shift. A developmental study revealed that infants showed joint attention behaviours, not only for the eyes, but also for the hand-pointing gestures (Csibra, 2003). An experimental study in adults showed that hand-pointing gestures were processed automatically (Langton \& Bruce, 2000). The researchers used the interference effect of stimulus presentation on the processing of spoken directional words. They reported an automatic interference effect induced by the hand-pointing presentation. These data suggest that hand pointing may trigger attentional shift in the same automatic manner as the eyes apparently do, although to date no studies tested this issue.

Here, to test the special status of eyes as automatic attention-triggering stimuli, we investigated whether and how the automatic attentional shift could be triggered by eyes, hand gestures, and arrows. We conducted experiments using a cuing paradigm. A nonpredictive cue of eyes, a hand, or an arrow was presented centrally before a target was presented peripherally. Photographic stimuli were used, as in previous studies (e.g., Driver et al., 1999). Only the arrow cue was a pictorial stimulus (Fig. 1). The participants were asked to localize the target as quickly as possible. By preparing multiple stimulus onset asynchrony (SOA) conditions, we analysed the time course of attentional shift triggered by these stimuli. Based on evidence suggesting that the automatic attentional shift could be triggered by all of these stimuli, we hypothesized that eyes, hands, and arrows trigger attentional shift in a similar manner. 


\section{Eyes}

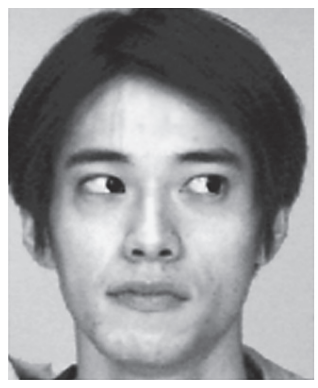

Hand

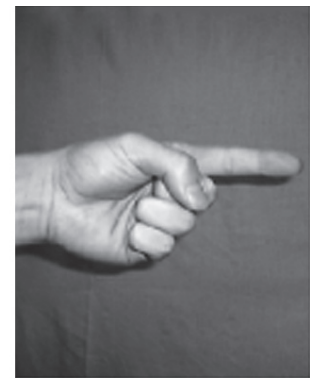

Arrow

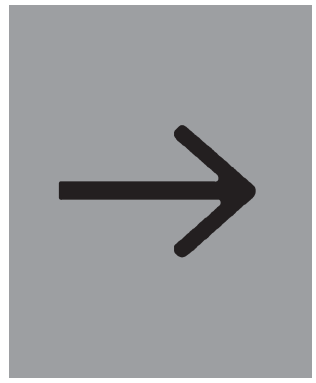

Fig. 1. Examples of cues in Experiment 1.

\section{EXPERIMENT 1}

In Experiment 1, we contrasted the cue-target relations of valid (i.e., the gaze direction was towards the target; left-left or right-right) versus invalid (i.e., the gaze direction was away from the target; left-right or right-left) conditions. We predicted that valid cues would shorten the RT to localize the targets compared with invalid cues for all three types of stimuli with similar temporal profiles.

\section{METHODS}

\section{Participants}

Fourteen healthy volunteers ( 3 women and 11 men; mean age, 24.3 years) participated in the experiment. All participants were right-handed and had normal or corrected-to-normal visual acuity. Informed consent was obtained from all participants in written form after the experimental procedures had been fully explained.

\section{Experimental Design}

The experiment was constructed as a within-subject three-factorial design, with stimulus type (eyes/ hand/arrow), cue-target validity (valid/invalid), and SOA (100/300/1000 ms) as factors.

\section{Apparatus}

The events were controlled by SuperLab Pro 2.0 (Cedrus) implemented on a Windows computer (MA55J, NEC). The stimuli were presented on a 19-inch flat-type CRT monitor (UltraScan P991, Dell) with a refresh rate of $100 \mathrm{~Hz}$ and a resolution of $1024 \times 768$ pixels. The participants' responses were recorded using a response box (RB-400, Cedrus).

\section{Stimuli}

The cues directing either to the left or right were indicated by eyes, a hand, or an arrow (Fig. 1). For eye cues, a gray-scale photograph of a full-face neutral expression of a man was prepared. To change the eye direction of the stimulus to a person looking left, the positions of his pupils were horizontally shifted using Photoshop 6.0 (Adobe). The mirror image of this photo was created as the cue indicating right. For hand cues, a gray-scale photograph of a hand indicating to left was prepared. The mirror image of the photo was used as the cue indicating right. The arrow cues consisted of a horizontal line subtending $6.0^{\circ}$ horizontally and two oblique lines, which tilted $45^{\circ}$ from the horizontal line and subtended $3.5^{\circ}$. The width of the line was 
$0.5^{\circ}$. The mirror images of the arrow cue were used as the cues indicating left and right. All of these stimuli were depicted in a rectangle on a gray plane background, subtending $10.2^{\circ}$ vertical $\times 13.1^{\circ}$ horizontal. The mean luminance of all images was adjusted to be the same using MATLAB 6.0 (Mathworks). The target was an open circle subtending $1.0^{\circ}$ vertically $\times 1.0^{\circ}$ horizontally.

Procedure

The experiments were conducted individually in a soundproof room. The participant was seated comfortably with her/his head supported by a chin-and-forehead rest, $0.57 \mathrm{~m}$ from the monitor.

The participants completed a total of 540 trials. A break was interposed after each of 180 trials. Trials were presented in pseudo-random order. At the beginning of the experiment, participants received 15 practice trials.

In each trial, a fixation point was presented centrally for $680 \mathrm{~ms}$ and a cue was then presented at the center of the screen for 100,300 , or $1000 \mathrm{~ms}$. After the cue disappeared, a target was presented in either the left or right visual field $\left(8.5^{\circ}\right.$ from the center) until a response was made.

As in previous studies (e.g., Friesen \& Kingstone, 1998), participants were instructed to localize whether targets appeared on the left or right side of the monitor as quickly as possible. Participants were told that the stimuli preceding the targets were not predictive. The response for the left or right target was made by pressing the key on the response box using the left or right index fingers, respectively. The time from the target onset to the response was recorded as RT.

\section{Data Analysis}

The data were analyzed using SPSS 10.0J (SPSS Japan). Mean RT of correct responses was calculated for each condition, excluding measurements beyond the mean $\pm 2 S D$ as artifacts $(<3.5 \%)$. The RT was analyzed using a 3 (cue type) $\times 2$ (cue-target validity) $\times 3$ (SOA) repeated-measures ANOVA. As confirmation, separate ANOVAs were conducted to test simple main effects of cue-target validity. In cases in which the assumption of sphericity was not met ( $p<.1$, Mauchley's sphericity test), the Greenhouse-Geisser adjusted degree of freedom was used.

To confirm that the RT data were not explained by a speed-accuracy trade-off phenomenon, the numbers of errors were also analyzed using repeated-measures ANOVA of the same design used in the RT analysis.

The results of statistical tests were deemed statistically significant at $p<.05$. Based on our preliminary analyses, the gender and age of the participants and the target position, which showed no significant effects on the results, were ignored in the analyses.

\section{RESULTS}

$R T$

The results of RT are shown in Fig. 2. The 3 (cue type) $\times 2$ (cue-target validity) $\times 3$ (SOA) repeated-measures ANOVA for the RT revealed significant main effect of cuetarget validity, which indicated that RTs were shorter for valid than for invalid cues, $F(1$, $13)=29.77, p<.001, \eta_{\mathrm{p}}^{2}=.660$. The main effect of SOA was also significant, $F(2$, $26)=10.51, p<.001, \eta_{\mathrm{p}}{ }^{2}=.369$. There was no other significant main effect or interaction, $p \mathrm{~s}>.1$. Separate ANOVAs confirmed that simple main effects of cue-target validity were significant for all cue type conditions, $F_{\mathrm{S}}(1,13)=13.63,31.07$, and 9.62, $p \mathrm{~s}<.005, .001$, and $.01, \eta_{\mathrm{p}}{ }^{2}=.532, .721$, and .445 for eyes, hand, and arrow, respectively.

\section{Error}

The mean $( \pm S D)$ percentage of total errors was $0.98( \pm 0.99) \%$. For the number of errors, the ANOVA revealed no significant main effects or interactions, $p \mathbf{s}>.1$. These results suggest that the RT-accuracy trade-off does not explain the RT results. 


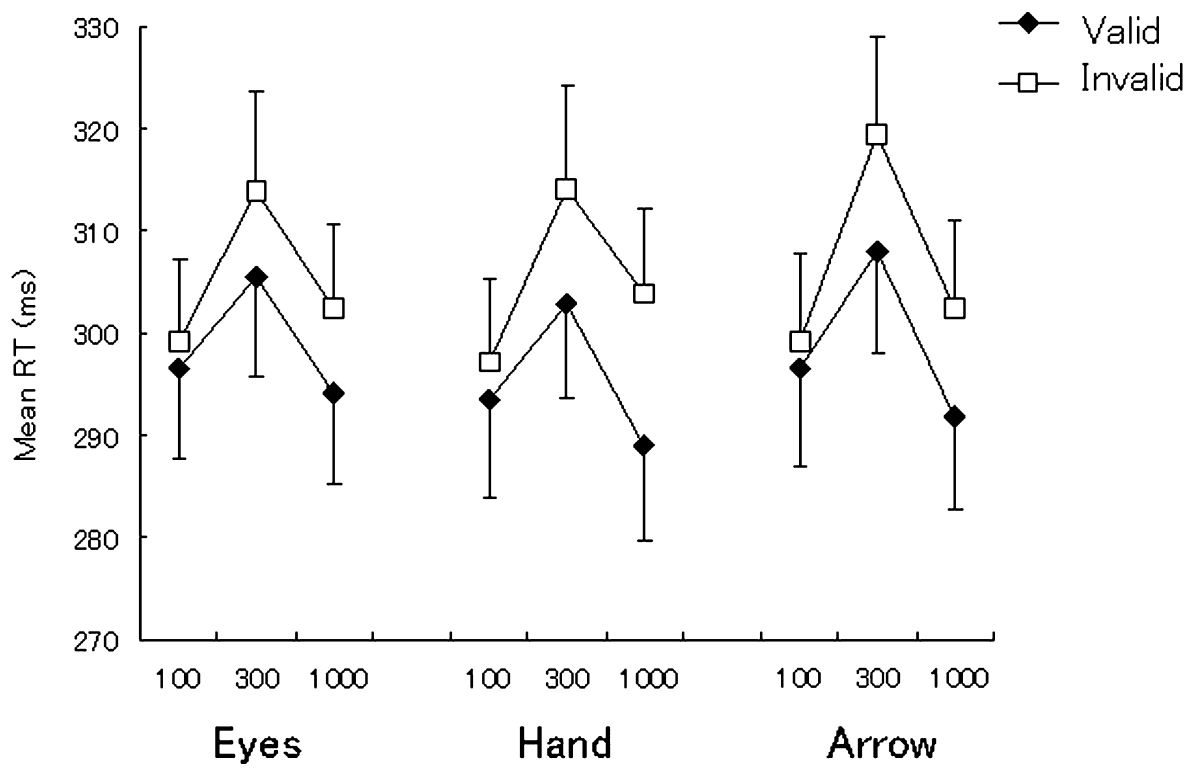

Fig. 2. Mean (with SE) reaction time (RT) in Experiment 1.

\section{DISCUSSION}

The eyes, a hand, and an arrow induced automatic attentional shift. The common pattern of time courses indicate that all of the cue types consistently induced rapid and sustained attentional shift. The results for eyes and arrows are consistent with those of previous studies using eyes (e.g., Driver et al., 1999) and arrows (e.g., Hommel et al., 2001). The result for hands is the first evidence, but an automatic process for handpointing gestures agrees with the results of a previous study that used a different paradigm (Langton \& Bruce, 2000). These results support our hypothesis that eyes, hands, and arrows can trigger a similar pattern of attentional shift.

\section{EXPERIMENT 2}

In the above experiment, we contrasted valid versus invalid cue-target relations to simplify the experimental design. Because of this design, it remained unclear whether the effect was a facilitating effect of valid cues, an interfering effect of invalid cues, or both. To evaluate these possibilities, we introduced a neutral condition in the cue-target validity factor (Fig. 3). Because there was no systematic difference in the temporal profiles of RT among stimulus types in Experiment 1, only $300 \mathrm{~ms}$ SOA was used. Based on previous findings of both facilitative and inhibitory effects using gaze cues (e.g., Hietanen, 1999) and the results of Experiment 1, which indicated similar patterns across stimulus types, we predicted that valid and invalid cues would shorten and lengthen the RT, respectively, compared to neutral cues, similarly for eyes, hands, and arrows. 


\section{Eyes}

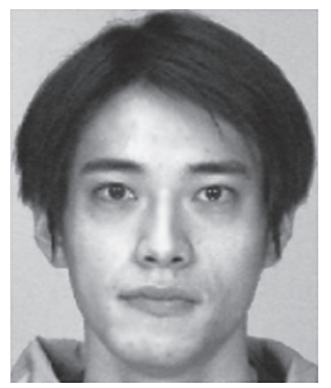

Hand

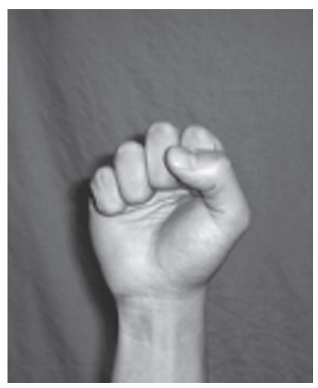

Arrow

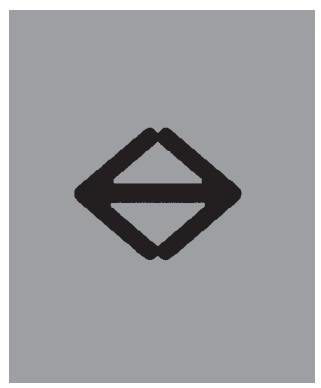

Fig. 3. Examples of cues in Experiment 2.

\section{METHODS}

\section{Participants}

Ten healthy volunteers (all men; mean age, 22.3 years) participated in this experiment. All participants were right-handed and had normal or corrected-to-normal visual acuity. Informed consent was obtained from all participants in written form after the experimental procedures had been fully explained.

\section{Experimental Design}

The experiment was constructed as a within-subject two-factorial design, with stimulus type (eyes/ hand/arrow), cue-target validity (valid/neutral/invalid) as factors.

Apparatus

The apparatus were identical to those used in Experiment 1.

Stimuli

In addition to the stimuli used in Experiment 1, we prepared cues that were neutral for direction (Fig. 3). For an eyes cue, a gray-scale photograph of a full-face neutral face of the stimulus person gazing straight ahead was prepared. For a hand cue, a gray-scale photograph of a fist was prepared. For an arrow cue, a lozenge was constructed using the same horizontal and oblique lines used in Experiment 1. As in Experiment 1 , the stimuli were depicted in rectangle on a gray plane background subtending $10.2^{\circ}$ vertical $\times 13.1^{\circ}$ horizontal, and their mean luminance was adjusted to be identical. As in Experiment 1, the target was an open circle subtending $1.0^{\circ}$ vertically $\times 1.0^{\circ}$ horizontally.

\section{Procedure}

The procedure was identical with that in Experiment 1 except for three alterations. First, only the SOA of $300 \mathrm{~ms}$ was used. Second, the neutral cue direction was added. Third, the number of trials was changed. The participants completed a total of 270 trials, with a break after 135 trials. Trials were presented in pseudorandom order.

\section{Data Analysis}

The data analysis was the same as that used in Experiment 1, except for two changes. First, a 3 (cue type) $\times 3$ (cue-target validity) repeated-measures ANOVA was conducted. Second, for significant effects of cue-target validity, follow-up multiple comparisons were conducted with Bonferroni's correction. As in Experiment 1, preliminary analyses showed no significant effects of the age of participants and the target position, and hence these factors were disregarded in the following analyses. 


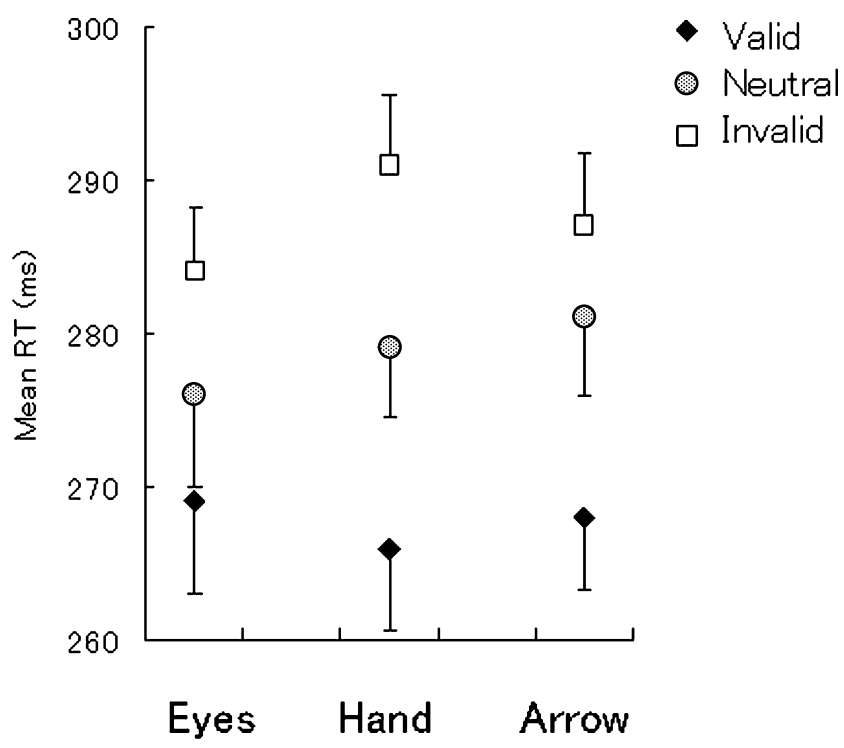

Fig. 4. Mean (with SE) reaction time (RT) in Experiment 2.

\section{RESULTS}

$R T$

The results of RT are shown in Fig. 4. The 3 (cue type) $\times 3$ (cue-target validity) repeated-measures ANOVA for the RT only revealed significant main effect of cue-target validity, $F(2,18)=24.38, p<.05, \eta_{\mathrm{p}}{ }^{2}=.735$. There were no other significant main effect or interaction, $p s>.1$. Follow-up multiple comparisons for the main effect of cue-target validity indicated that the RT for the valid condition was shorter than that for either neutral or invalid conditions, and the RT for the neutral condition was shorter than that for the invalid condition, $t \mathrm{~s}(9)=5.68$ and $3.90, p \mathrm{~s}<.001$ and $.005, d \mathrm{~s}=.903$ and .688 for valid vs neutral and invalid vs neutral conditions, respectively. Separate ANOVAs confirmed that simple main effects of cue-target validity were significant for all cue type conditions, $F_{\mathrm{s}}(2,18)=16.24,13.72$, and 17.33, all $p \mathrm{~s}<.001, \eta_{\mathrm{p}}{ }^{2}=.643, .604$, and .658 for eyes, hand, and arrow, respectively.

\section{Error}

The mean $( \pm S D)$ percentage of total errors was $0.85( \pm 0.91) \%$. For the number of errors, the ANOVA revealed no significant main effects or interactions, $p \mathrm{~s}>.1$. These results suggest that the RT-accuracy trade-off does not explain the RT results. 


\section{DISCUSSION}

Our results revealed that valid and invalid cues facilitated and interfered target localizations, respectively, than did neutral cues for eyes, hands, and arrows. These results are consistent with those of previous studies (e.g., Hietanen, 1999; Hommel et al., 2001), and support our hypothesis that eyes, hands, and arrows could automatically trigger a similar pattern of attentional shift.

\section{GENERAL DisCUSSION}

Our results consistently showed that the eyes, a hand, and an arrow automatically induced attentional shift in a similar pattern. These results suggest that a common psychological mechanism is involved in the automatic attentional shift by gaze, gestures, and symbols. These results are consistent with human developmental studies indicating that infants follow the direction of attention of adults, which were indicated by eyes or hand-pointing gestures (Csibra, 2003). An animal study also indicated that chimpanzees showed automatic attentional shifting while viewing arrows (Itakura, 2001). These developmental and comparative data suggest that a shared psychological mechanism in human beings for the automatic attentional shift in response to gaze, gestures, and some kinds of symbols may have developed through the evolutionary process.

We speculate that the neural substrate of the present common attentional shift may include the posterior superior temporal sulcus (STS). Some previous neuroimaging studies described the involvement of the posterior STS in the processing of eye gaze (e.g., Hoffman \& Haxby, 2000) and hand gestures (e.g., Grezes, Costes, \& Decety, 1999). Furthermore, a meta-analysis of neuroimaging data showed shared cortical regions, including the posterior STS and its adjacent region, for covert shifts of attention and gaze perception (Grosbras, Laird, \& Paus, 2005). These data suggest that a common neural mechanism, which includes the posterior STS, may implement the automatic attentional shift triggered by gaze, gestures, and symbols.

We should point out that some previous studies have reported conflicting results regarding attentional orienting in response to gaze and symbolic cues. For example, Friesen et al. (2004) did not find automatic attention orienting for the viewing of arrow cues. The differences may be partially explained by top-down modulation of stimulusdriven automatic attentional shift. A previous study used ambiguous cues that could be perceived either as representing eyes or cars in a cueing-paradigm (Ristic \& Kingstone, 2005). Although the stimuli elicited automatic attentional shifts when they were referred to as eyes, they did not do so when referred to as cars. These data suggest that the automatic attention orienting process could be modulated by top-down cognitive strategies. In future research, it would be interesting to manipulate the cognitive strategies of participants while viewing gaze, gestures, and symbols.

Promising directions for future studies include the investigation of the coordination among automatic attentional shifts triggered by gaze, gestures, and symbols. For 
example, in everyday life, we combine gaze and hand-pointing gestures for having others attend to given locations. Previous studies showed that hand pointing and gaze were directed to the same targets (Gielen, van den Heuvel, \& van Gisbergen, 1984), and handpointing gestures occurred briefly after gaze movements (Prablanc, Echaller, Komilis, \& Jeannerod, 1979). It may be possible that hand pointing gestures augment automatic attentional shifts triggered by gaze.

In summary, our results indicated that non-predictive cues, which were expressed by eyes, hands, or arrows, had similar effects on the RT in localizing the targets. The results suggest a common psychological mechanism for the automatic attentional shift triggered by gaze, gestures, and symbols.

\section{REFERENCES}

Csibra, G. 2003. Teleological and referential understanding of action in infancy. Philosophical Transactions of the Royal Society of London Series B: Biological Sciences, 358, 447-458.

Driver, J., Davis, G., Ricciardelli, P., Kidd, P., Maxwell, E., \& Baron-Cohen, S. 1999. Gaze perception triggers reflexive visuospatial orienting. Visual Cognition, 6, 509-540.

Emery, N. J., Lorincz, E. N., Perrett, D. I., \& Oram, M. W. 1997. Gaze following and joint attention in rhesus monkeys (Macaca mulatta). Journal of Comparative Psychology, 111, 286-293.

Friesen, C. K., \& Kingstone, A. 1998. The eyes have it! Reflexive orienting is triggered by nonpredictive gaze. Psychonomic Bulletin and Review, 5, 490-495.

Friesen, C. K., Ristic, J., \& Kingstone, A. 2004. Attentional effects of counterpredictive gaze and arrow cues. Journal of Experimental Psychology: Human Perception and Performance, 30, 319-329.

Frischen, A., Bayliss, A. P., \& Tipper, S. P. 2007. Gaze cueing of attention: Visual attention, social cognition, and individual differences. Psychological Bulletin, 133, 694-724.

Gielen, C. C., van den Heuvel, P. J., \& van den Gisbergen, J. A. 1984. Coordination of fast eye and arm movements in a tracking study. Experimental Brain Research, 56, 154-161.

Grezes, J., Costes, N., \& Decety, J. 1999. The effects of learning and intention on the neural network involved in the perception of meaningless actions. Brain, 122, 1875-1887.

Grosbras, M. H., Laird, A. R., \& Paus, T. 2005. Cortical regions involved in eye movements, shifts of attention, and gaze perception. Human Brain Mapping, 25, 140-154.

Hietanen, J. K. 1999. Does your gaze direction and head orientation shift my visual attention? Neuroreport, 10, 3443-3447.

Hoffman, E. A., \& Haxby, J. V. 2000. Distinct representations of eye gaze and identity in the distributed human neural system for face perception. Nature Neuroscience, 3, 80-84.

Hommel, B., Pratt, J., Colzato, L., \& Godijn, R. 2001. Symbolic control of visual attention. Psychological Science, 12, 360-365.

Itakura, S. 2001. Visual attention following: From exogenously to endogenously. In Brookfield Zoo (Ed.), The apes: Challenges for the 21st Century (pp. 372). Brookfield, Ill: Chicago Zoological Society.

Langton, S. R. H., \& Bruce, V. 2000. You must see the point: Automatic processing of cues to the direction of social attention. Journal of Experimental Psychology: Human Perception and Performance, 26, 747-757.

Posner, M. I. 1980. Orienting of attention. Quarterly Journal of Experimental Psychology, 32, 3-25.

Prablanc, C., Echallier, J. F., Komilis, E., \& Jeannerod, M. 1979. Optimal response of eye and hand motor systems in pointing at a visual target. I. Spatiotemporal characteristics of eye and hand movements and their relationships when varying the amount of visual information. Biological Cybernetics, 35, 113-124.

Ristic, J., Friesen, C. K., \& Kingstone, A. 2002. Are eyes special? It depends on how you look at it. Psychonomic Bulletin and Review, 9, 507-513.

Ristic, J., \& Kingstone, A. 2005. Taking control of reflexive social attention. Cognition, 94, B55-B65.

Sato, W., Okada, T., \& Toichi, M. 2007. Attentional shift is triggered by gaze without awareness. Experimental Brain Research, 183, 87-94.

Tipples, J. 2002. Eye gaze is not unique: automatic orienting in response to uninformative arrows. Psychonomic Bulletin and Review, 9, 314-318. 